\title{
Clinical Utility of Chest Computed Tomography in Patients with Rib Fractures CT Chest and Rib Fractures
}

\author{
Brandon C. Chapman, ${ }^{1, *}$ Douglas M. Overbey, ${ }^{1}$ Feven Tesfalidet, ${ }^{2}$ Kristofer Schramm, ${ }^{3}$ Robert T. \\ Stovall, ${ }^{4}$ Andrew French, ${ }^{5}$ Jeffrey L. Johnson, ${ }^{4}$ Clay C. Burlew, ${ }^{4}$ Carlton Barnett, ${ }^{4}$ Ernest E. Moore, ${ }^{4}$ and
} Fredric M. Pieracci ${ }^{4}$

${ }^{1}$ Department of Surgery, University of Colorado School of Medicine, Colorado, United States

${ }^{2}$ School of Medicine, University of Colorado, Colorado, United States

${ }^{3}$ Department of Radiology, School of Medicine, University of Colorado, Colorado, United States

${ }^{4}$ Department of Surgery, Denver Health Medical Center, Colorado, United States

${ }^{5}$ Department of Emergency Medicine, Denver Health Medical Center, Denver, CO, United States

"Corresponding author: Brandon C. Chapman, Department of Surgery, University of Colorado School of Medicine, Colorado, United States. Tel: +1-7207373858, E-mail: brandon.chapman@ucdenver.edu

Received 2016 February 12; Revised 2016 May 10; Accepted 2016 September 04.

\begin{abstract}
Background: Chest CT is more sensitive than a chest X-ray (CXR) in diagnosing rib fractures; however, the clinical significance of these fractures remains unclear.

Objectives: The purpose of this study was to determine the added diagnostic use of chest CT performed after CXR in patients with either known or suspected rib fractures secondary to blunt trauma.

Methods: Retrospective cohort study of blunt trauma patients with rib fractures at a level I trauma center that had both a CXR and a CT chest. The CT finding of $\geq 3$ additional fractures in patients with $\leq 3$ rib fractures on CXR was considered clinically meaningful. Student's t-test and chi-square analysis were used for comparison.

Results: We identified 499 patients with rib fractures: 93 (18.6\%) had CXR only, 7 (1.4\%) had chest CT only, and 399 (79.9\%) had both CXR and chest CT. Among these 399 patients, a total of 1,969 rib fractures were identified: 1,467 (74.5\%) were missed by CXR. The median number of additional fractures identified by CT was 3 (range, 4 -15). Of 212 (53.1\%) patients with a clinically meaningful increase in the number of fractures, 68 patients underwent one or more clinical interventions: 36 SICU admissions, 20 pain catheter placements, 23 epidural placements, and 3 SSRF. Additionally, 70 patients had a chest tube placed for retained hemothorax or occult pneumothorax. Overall, 138 patients (34.5\%) had a change in clinical management based upon CT chest.

Conclusions: The chest X-ray missed $\sim 75 \%$ of rib fractures seen on chest CT. Although patients with a clinical meaningful increase in the number of rib fractures were more likely to be admitted to the intensive care unit, there was no associated improvement in pulmonary outcomes.
\end{abstract}

Keywords: Rib Fractures, Tomography X-Ray Compute, X-rays, Thoracic Injuries.

\section{Background}

Rib fractures are present in approximately $10 \%$ of trauma patients and are a marker of injury severity. Among patients with rib fractures, 90\% will have associated injuries, 50\% will require operative and intesive care unit care, 33\% will develop pulmonary complications, 33\% will require discharge to an extended care facilty, and $12 \%$ will die before hopsital discharge (1). The number of rib fractures correlates exponentially with both morbidity and mortality (2-4). For each additional rib fracture in patients age 65 or older, the risk of pneumonia increases by $27 \%$ and risk of death by $19 \%$ (5).
Because of the significant morbidity and mortality associated with rib fractures, it is imperative that these highrisk patients are identified. Chest X-ray (CXR) is routinely obtained in blunt trauma patients on arrival in the emergency department to evaluate for thoracic trauma. Although CXR is a rapid means by which to rule out immediately life-threatening injuries, more subtle injuries are frequently missed. Computed tomography (CT) of the chest has been shown to identify many injuries that were otherwise missed by CXR including pulmonary contusions, hemothorax or pneumothorax, and rib fractures (6-10). However, it is unclear if these additional injuries identified 
on CT chest will change clinical management and impact patient outcomes.

\section{Objectives}

The purpose of this study was to determine if chest CT performed after CXR in patients with rib fractures secondary to blunt trauma will result in a change in clinical management and outcomes. We hypothesized that chest CT will identify significantly more rib fractures than CXR and will result in a change in clinical management reducing clinical outcomes.

\section{Methods}

We performed a retrospective cohort study of all patients sustaining blunt trauma at Denver Health Medical Center, an American College of Surgeons-verified, state certified Level I trauma center, from September 2012 to April 2014. The study protocol conforms to the ethical guidelines of the 1975 Declaration of Helsinki and was approved by the Colorado Institional Review Board. We identified all patients who were diagnosed with one or more rib fractures who underwent both a CXR and a contrast-enhanced spiral CT of the chest. Although at the discretion of the emergency department physician and trauma surgeon, CT chest is typically performed at our institution based on the mechanism of injury: high-energy deceleration motor vehicle collision with frontal or lateral impact ( $>30 \mathrm{mph}$ frontal impact and $>23 \mathrm{mph}$ lateral impact), motor vehicle collision with ejection, falls of $>25$ feet, or direct impact (horse kick to the chest, snowmobile, or ski collision with tree) (11, 12).

Patient demographics including age, gender, mechanism of injury (fall, MVC/motorcycle collision autopedestrian, other), and injury severity score (ISS) were recorded. Radiology reports read by board certified radiologists were reviewed for the number of rib fractures, effusion/hemothorax, pneumothorax and pulmonary contusion, presence or absence of chest tube, and other associated injuries. We defined a chest CT finding of $\geq 3$ additional fractures in patients with $\leq 3$ rib fractures on CXR as clinically meaningful because we asssumed that this would result in an escalation of care i.e., admission to hospital, admission to surgical intensive care unit (SICU), insertion of pain catheter or epidural, and/or surgical stabilization of rib fractures (SSRF).

We reviewed the medical record for management decisions including SICU, placement of paravertebral percutenaous analgesic catheters including intercostal pain catheters and epidural, had a chest tube placed for pneumothorax and/or hemothorax, or underwent SSRF. We defined a change in clincial management in patients who had radiologic clinically meaningful findings and underwent one or more of the following interventions: admitted to the SICU, insertion of pain catheter or epidural, and SSRF. Admission to the SICU was considered a change in management only in patients with isolated rib fractures and no other indication for admission to the intensive care unit. In addition, we considered placement of a chest tube in a patient with one or more rib fractures for an occult pneumothorax or retained hemothorax as a change in clinical management.

Clinical outcomes including acute respiratory failure, pneumonia (defined by clinical symptoms with culture of bronchoalveolar lavage $>10^{5} \mathrm{cfu} / \mathrm{mL}$ ), acute respiratory distresss sydnrome, tracheostomy, overall hospital length of stay, SICU length of stay, time on mechanical ventilation, and overall mortality were recorded.

Statistical analyses were performed with SAS version 9.0 (SAS Inc., Carey, NC). Data are listed as mean (range) or number (\%). Continious variables were compared using the student's t-test, and categorical veriables were compared using the chi squared test. Statistical significancce was defined as $\mathrm{P}<0.05$.

\section{Results}

We identified 499 patients with rib fractures: 93 (18.6\%) had CXR only, 7 (1.4\%) had chest CT only, and 399 (79.9\%) had both CXR and chest CT (Figure 1). Among the 399 patients who had both CXR and chest CT, a total of 1,969 rib fractures were identified and 1,467 (74.5\%) were missed by CXR. The median number of fractures on CXR was 0 (range 0 -13) and on chest CT 4 (range 1-18). The median number of additional fractures identified by CT compared to CXR was 3 (range -4 - 15) (Figure 2). A negative number indicated that CXR overcalled the number of rib fractures seen on chest CT. The location of the rib fractures are listed in Table 1.

A clinically meaningful increase in the number of rib fractures identified by chest CT was found in 212 of 399 patients (53.1\%) with both a chest CT and CXR (Table 2). In this subgroup, 68 patients underwent one or more clinical interventions: 36 SICU admissions, 20 pain catheter placements, 23 epidural placements, and 3 SSRF (Figure 1). This represented $32 \%$ of the clinically meaningful group and $17 \%$ of the total sample. Fourteen patients underwent 2 or more interventions (e.g., admitted to the SICU and had a pain catheter placed). Of the 399 patients, 70 patients had a chest tube placed for retained hemothorax or occult pneumothorax. In total, 138 patients (34.5\%) had a change in clin- 


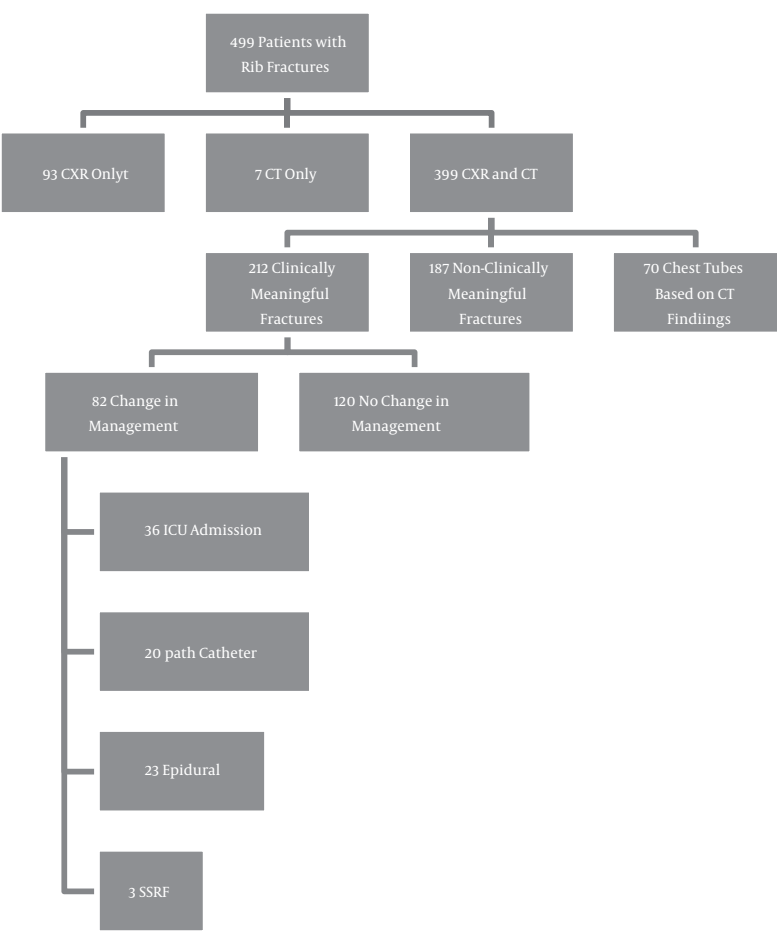

Figure 1. Flow Chart of Patients Enrolled in Study

Table 1. Number of Patients With Corresponding Rib Fracture Location Among All 399 Patients Undergoing CXR and CT Chest

\begin{tabular}{lcccc}
\hline & No Fractures & Right Fracture & Left Fracture & Bilateral Fracture \\
\hline CXR & $245(61.4)$ & $45(11.3)$ & $60(15.0)$ & $149(37.4)$
\end{tabular}

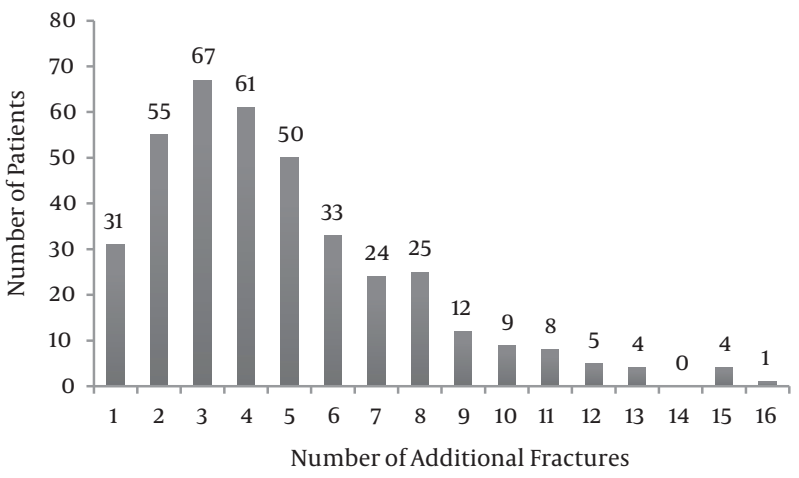

Figure 2. Number of Additional Rib Fractures Seen on Chest CT Compared to CXR

ical management(SICU admission, pain catheter, epidural, SSRF, chest tube) based upon CT chest.

As compared to patients without a clinically significant increase in the number of rib fractures, patients with a clinically significant increase were more likely to be older $(\mathrm{P}=$ $0.01)$ and have a greater number of rib fractures $(\mathrm{P}<0.01)$. Patients in both groups had similar clinical outcomes and interventions, with the exception of the clinically meaningful group being more likely to be admitted to the SICU $(\mathrm{P}<0.01)$ (Table 2).

\section{Discussion}

Current evidence suggests that chest CT identifies additional thoracic injuries including rib fractures, pneumothorax, pulmonary contusions, and major vascular injury in $39 \%-50 \%$ of patients presenting with blunt trauma $(7,9$, 10). However, routine chest CT it is not without risk to the patient: it is costly, requires radiation exposure, and removes the patient briefly from direct clinical care at a time when close monitoring of the patient is critical. Perhaps most importantly, additional findings on CT scan may 
Table 2. Patient Demographics, Clinical Outcomes, and Clinical Interventions Among Patients With and Without a Clinically Meaningful Increase in the Number of Rib Fractures on Chest $\mathrm{CT}^{\mathrm{a}}$

\begin{tabular}{|c|c|c|c|c|}
\hline & All Patients $(n=399)$ & Clinically Meaningful $(n=212)$ & Not Clinically Meaningful $(n=187)$ & P Value \\
\hline \multicolumn{5}{|l|}{ Patient Demographics } \\
\hline Age, $y$ & $47.9(4-101)$ & $50.3(4-101)$ & $45.4(13-95)$ & 0.01 \\
\hline Male & $114(28.6)$ & $55(25.9)$ & $59(31.6)$ & 0.22 \\
\hline Mechanism & & & & 0.20 \\
\hline Fall & $81(20.3)$ & $47(22.2)$ & $34(18.2)$ & \\
\hline MVC/MCC & $216(54.1)$ & $115(54.3)$ & $101(54.0)$ & \\
\hline Auto pedestrian & $54(13.5)$ & $22(10.4)$ & $32(17.1)$ & \\
\hline Other & $48(12.1)$ & $28(13.2)$ & $20(10.7)$ & \\
\hline Injury Severity Score & $19.6(3-66)$ & $19.5(9-66)$ & $19.7(3-57)$ & 0.81 \\
\hline Ribs fractured & $4.9(1-18)$ & $6(3-15)$ & $4(1-18)$ & $<0.01$ \\
\hline \multicolumn{5}{|l|}{ Clinical Outcomes } \\
\hline ARF & $133(33.3)$ & $67(31.6)$ & $66(35.3)$ & 0.44 \\
\hline Pneumonia & $53(13.2)$ & $31(14.6)$ & $22(11.8)$ & 0.40 \\
\hline ARDS & $6(1.5)$ & $3(1.4)$ & $3(1.6)$ & 0.88 \\
\hline Tracheostomy & $61(15.3)$ & $33(15.6)$ & $28(15.0)$ & 0.87 \\
\hline Hospital LOS (days) & $11.5(1-102)$ & $11.2(1-92)$ & $12.1(1-102)$ & 0.53 \\
\hline SICU LOS (hours) & $111.8(0-1,125)$ & $111.7(0-1,125)$ & $111.8(0-841)$ & 0.99 \\
\hline Ventilator minutes & $4407.3(0-64,919)$ & $4,324(0-64,919)$ & $4,502(0-60,584)$ & 0.85 \\
\hline Mortality & $12(3.0)$ & $8(3.8)$ & $4(2.1)$ & 0.34 \\
\hline \multicolumn{5}{|l|}{ Clinical Intervention } \\
\hline SICU admission & $36(9.0)$ & $36(9.0)$ & 0 & $<0.01$ \\
\hline Pain catheter & $40(10.0)$ & $20(9.4)$ & $20(10.7)$ & 0.68 \\
\hline Epidural & $38(38.1)$ & $23(10.8)$ & $15(8.0)$ & 0.27 \\
\hline SSRF & $10(2.5)$ & $3(1.4)$ & $7(3.7)$ & 0.26 \\
\hline
\end{tabular}

Abbreviations: ARDS, acute respiratory distress syndrome; ARF, acute respiratory failure; MCC, motorcycle collision; MVC, motor vehicle collision; LOS, length of stay; SICU, surgical intensive care unit; SSRF, surgical stabilization of rib fractures.

${ }^{\mathrm{a}}$ Mean (range) unless otherwise stated.

not result in a change in patient care management. Some studies have found that chest CT after a normal CXR rarely detects a clinically significant injury (13-15), whereas other have shown a change in clinical management in 17\% - 30\% of patients $(6,8-10)$.

In the current study, 138 patients (34.5\%) had a change in clinical management based upon chest CT. Among patients with a clinically meaningful increase in the number of rib fractures, 68 patients underwent one or more clinical interventions: 36 SICU admissions, 20 pain catheter placements, 23 epidural placements, and 3 SSRF. An additional 70 patients had a chest tube placed for retained hemothorax or occult pneumothorax. However, there was no improvement in pulmonary morbidity (acute respiratory failure, duration of mechanical ventilation, pneumonia, acute respiratory distress syndrome, need for tracheostomy), hospital length of stay, SICU length of stay, and mortality among patients with a clinically meaningful increase in the number of rib fractures. This finding may be a reflection of our definition of a clinically meaningful in- crease in the number of rib fractures: chest CT finding of $\geq 3$ additional fractures in patients with $\leq 3$ rib fractures on CXR, i.e., patients without a clinically meaningful increase may have 3 or more rib fractures seen on both CXR and CT and both groups were treated similarly with aggressive pulmonary toilet in the SICU, pain control with pain catheters or epidurals, and SSRF.

Although outcomes were similar in the present study, chest CT provides detailed anatomic fracture patterns that may predict pulmonary outcomes. At our institution, we developed the RibScore, which is a radiographic rib fracture scoring system that incorporates 6 variables seen on CT chest: (1) six or more ribs fractured, (2) bilateral rib fractures, (3) flail chest, (4) three or more severely (bicortical) displaced fractures, (5) first rib fracture, and (6) at least one fracture in each of the three anatomic areas (anterior, lateral, and posterior). The composite RibScore was significantly and linearly correlated with pneumonia, respiratory failure, and tracheostomy (16). There are several potential advantages of using chest CT that includes 
more detailed anatomic information regarding the fracture pattern. The location of the fractures (both in a superior to inferior direction and medial to lateral direction) may influence the decision to pursue locoregional analgesia with a thoracic epidural catheter as opposed to a percutaneous paraspinous catheter (17). Furthermore, the decision to perform surgical stabilization of rib fractures (SSRF) is based to some degree on detailed fracture information, such as flail chest and degree of displacement (18).

The present study does have several limitations. The data was obtained retrospectively and limited data collection variables. Not all trauma patients admitted to our trauma center undergo chest imaging and this discretion is left to the individual surgeon and may have resulted in a selection bias. Additionally, we assumed a change in management was based upon the number of additional rib fractures seen on CT chest. Furthermore, because all patients underwent both CXR and chest CT, we were not able to perform a cost analysis to determine whether chest CT was associated with an increased cost.

In conclusion, CXR missed approximately $75 \%$ of rib fractures seen on chest CT, and nearly half of these patients had a clinically meaningful increase in the number of rib fractures diagnosed. Although patients with a clinical meaningful increase in the number of rib fractures were more likely to be admitted to the intensive care unit, there was no associated improvement in clinical outcomes including length of stay, duration of mechanical ventilation, acute respiratory failure, pneumonia, acute respiratory distress syndrome, tracheostomy, and mortality.

\section{Footnote}

Authors' Contribution: Study concept and design: Brandon C. Chapman, Fredric M. Pieracci; acquisition of data: Brandon C. Chapman, Douglas M. Overbey, Feven Tesfalidet, Kristofer Schramm; analysis and interpretation of data: Brandon C. Chapman, Douglas M. Overbey, Feven Tesfalidet, Kristofer Schramm, Robert T. Stovall, Jeffery L. Johnson, Clay C. Burlew, Carlton Barnett, Ernest E. Moore, Fredric M. Pieracci; drafting of the manuscript: Brandon C. Chapman, Douglas M. Overbey, Fredric M. Pieracci; critical revision of the manuscript for important intellectual content: Brandon C. Chapman, Douglas M. Overbey, Robert T. Stovall, Jeffery L. Johnson, Clay C. Burlew, Carlton Barnett, Ernest E. Moore, Fredric M. Pieracci; statistical analysis: Brandon C. Chapman, Fredric M. Pieracci; administrative, technical, and material support: Brandon C. Chapman, Robert T. Stovall, Jeffery L. Johnson, Burlew, Carlton Barnett, Ernest E. Moore, Fredric M. Pieracci; study supervision: Brandon C. Chapman, Fredric M. Pieracci.

\section{References}

1. Ziegler DW, Agarwal NN. The morbidity and mortality of rib fractures. Journal of Trauma and Acute Care Surgery. 1994;37(6):975-9.

2. Flagel BT, Luchette FA, Reed RL, Esposito TJ, Davis KA, Santaniello JM, et al. Half-a-dozen ribs: the breakpoint for mortality. Surgery. 2005;138(4):717-23. doi: 10.1016/j.surg.2005.07.022. [PubMed: 16269301] discussion 723-5.

3. Holcomb JB, McMullin NR, Kozar RA, Lygas MH, Moore FA. Morbidity from rib fractures increases after age 45. J Am Coll Surg. 2003;196(4):549-55. doi: 10.1016/S1072-7515(02)01894-X. [PubMed: 12691929].

4. Lee RB, Bass SM, Morris JJ, MacKenzie EJ. Three or more rib fractures as an indicator for transfer to a Level I trauma center: a populationbased study.J Trauma. 1990;30(6):689-94. [PubMed: 2352298].

5. Bulger EM, Arneson MA, Mock CN, Jurkovich GJ. Rib fractures in the elderly.J Trauma. 2000;48(6):1040-6. [PubMed: 10866248] discussion 1046-7.

6. Brink M, Deunk J, Dekker HM, Kool DR, Edwards MJ, van Vugt AB, et al. Added value of routine chest MDCT after blunt trauma: evaluation of additional findings and impact on patient management. AJR Am J Roentgenol. 2008;190(6):1591-8. doi: 10.2214/AJR.07.3277. [PubMed: 18492911].

7. Exadaktylos AK, Sclabas G, Schmid SW, Schaller B, Zimmermann H. Do we really need routine computed tomographic scanning in the primary evaluation of blunt chest trauma in patients with "normal" chest radiograph?.J Trauma. 2001;51(6):1173-6. [PubMed: 11740271].

8. Guerrero-Lopez F, Vazquez-Mata G, Alcazar-Romero PP, FernandezMondejar E, Aguayo-Hoyos E, Linde-Valverde CM. Evaluation of the utility of computed tomography in the initial assessment of the critical care patient with chest trauma. Crit Care Med. 2000;28(5):1370-5. [PubMed: 10834680].

9. Omert L, Yeaney WW, Protetch J. Efficacy of thoracic computerized tomography in blunt chest trauma. Am Surg. 2001;67(7):660-4. [PubMed: 11450784].

10. Traub M, Stevenson M, McEvoy S, Briggs G, Lo SK, Leibman S, et al. The use of chest computed tomography versus chest X-ray in patients with major blunt trauma. Injury. 2007;38(1):43-7. doi: 10.1016/j.injury.2006.07.006. [PubMed: 17045268].

11. Dyer DS, Moore EE, Ilke DN, McIntyre RC, Bernstein SM, Durham JD, et al. Thoracic aortic injury: how predictive is mechanism and is chest computed tomography a reliable screening tool? A prospective study of 1,561 patients.JTrauma. 2000;48(4):673-82. [PubMed: 10780601] discussion 682-3.

12. Siegel JH, Smith JA, Siddiqi SQ. Change in velocity and energy dissipation on impact in motor vehicle crashes as a function of the direction of crash: key factors in the production of thoracic aortic injuries, their pattern of associated injuries and patient survival. A Crash Injury Research Engineering Network (CIREN) study.J Trauma. 2004;57(4):760-77. [PubMed: 15514530] discussion 777-8.

13. Kaiser M, Whealon M, Barrios C, Dobson S, Malinoski D, Dolich M, et al. The clinical significance of occult thoracic injury in blunt trauma patients. Am Surg. 2010;76(10):1063-6. [PubMed: 21105610].

14. Kea B, Gamarallage R, Vairamuthu H, Fortman J, Lunney K, Hendey $\mathrm{GW}$, et al. What is the clinical significance of chest CT when the chest $\mathrm{x}$-ray result is normal in patients with blunt trauma?. Am J Emerg Med. 2013;31(8):1268-73. doi: 10.1016/j.ajem.2013.04.021. [PubMed: 23796979].

15. Livingston DH, Shogan B, John P, Lavery RF. CT diagnosis of Rib fractures and the prediction of acute respiratory failure. J Trauma. 2008;64(4):905-11. doi: 10.1097/TA.0b013e3181668ad7. [PubMed: 18404055]. 
16. Chapman BC, Herbert B, Rodil M, Salotto J, Stovall RT, Biffl W, et al. RibScore: A novel radiographic score based on fracture pattern that predicts pneumonia, respiratory failure, and tracheostomy. J Trauma Acute Care Surg. 2016;80(1):95-101. doi: 10.1097/TA.0000000000000867. [PubMed: 26683395].

17. Truitt MS, Murry J, Amos J, Lorenzo M, Mangram A, Dunn E, et al. Continuous intercostal nerve blockade for rib fractures: ready for prime-
time?.J Trauma. 2011;71(6):1548-52. doi: 10.1097/TA.ob013e31823c96eo. [PubMed: 22182865] discussion 1552.

18. Pieracci FM, Rodil M, Stovall RT, Johnson JL, Biffl WL, Mauffrey C, et al. Surgical stabilization of severe rib fractures. J Trauma Acute Care Surg. 2015;78(4):883-7. doi: 10.1097/TA.0000000000000581. [PubMed: 25742255]. 